\title{
Synthesis and Crystal Structure of a Chain Complex of Rhodium(II) Acetate and 1,2-Bis(4-pyridyl)ethane having an $N_{2}$-Adsorption Property
}

\author{
Masahiro Mikuriya,*† Junya Yamamoto,* Kazuya Ouchi,* Shohei TaKada,* Daisuke Yoshioka,* \\ Hidekazu TANAKA, ** and Makoto HANDA***; \\ *Department of Chemistry and Research Center for Coordination Molecule-based Devices, School of Science \\ and Technology, Kwansei Gakuin University, 2-1 Gakuen, Sanda 669-1337, Japan \\ **Department of Chemistry, Interdisciplinary Graduate School of Science and Engineering, Shimane \\ University, 1060 Nishikawatsu, Matsue 690-8504, Japan
}

\begin{abstract}
The title compound, a chain adduct of rhodium(II) acetate with 1,2-bis(4-pyridyl)ethane (bpe), catena(tetrakis( $\mu$-acetao$\left.O, O^{\prime}\right)\left(\mu\right.$-1,2-bis(4-pyridyl)ethane- $\left.N, N^{\prime}\right)$ dirhodium(II)-water (1/2)), $\left[\mathrm{Rh}_{2}\left(\mathrm{CH}_{3} \mathrm{CO}_{2}\right)_{4}(\mathrm{bpe})\right]_{n} \cdot 2 n \mathrm{H}_{2} \mathrm{O}$, was isolated. The crystal structure was determined by the single-crystal X-ray diffraction method at $293 \mathrm{~K}$. It crystallizes in the monoclinic space group $C 2 / c$ with $a=24.937(5) \AA, b=13.211(3) \AA, c=8.8480(19) \AA, \beta=94.189(3)^{\circ}, V=2907.0(11) \AA^{3}, D_{\mathrm{x}}=1.513$ $\mathrm{g} / \mathrm{cm}^{3}$, and $Z=4$. The $R 1[I>2 \sigma(I)]$ and $w R 2$ (all data) values are 0.0356 and 0.0863 , respectively, for all 3324 independent reflections. The molecule has a linear chain with an alternating arrangement of $\mathrm{Rh}_{2}\left(\mathrm{CH}_{3} \mathrm{CO}_{2}\right)_{4}[\mathrm{Rh}-\mathrm{Rh}$ $2.3990(8) \AA]$ and bpe. The specific surface area estimated from a $\mathrm{N}_{2}$ adsorption isotherm was $65.6 \mathrm{~m}^{2} \mathrm{~g}^{-1}$.
\end{abstract}

(Received March 30, 2013; Accepted May 27, 2013; Published on web July 10, 2013)

There has been considerable interest in the molecular assembly of metal carboxylates with a lantern-like structure, because such complexes may have an adsorption property for small gas molecules. ${ }^{1-4}$ We have engaged in synthetic studies on rhodium(II) carboxylates with $N, N^{\prime}$-bidentate ligands to make molecular assembled systems. ${ }^{5-9}$ 1,2-Bis(4-pyridyl)ethane (bpe) is an interesting bidentate ligand used to make such compounds, because it has two binding sites with an ethane-type orientation. In this study, we isolated an adduct from a reaction of rhodium(II) acetate and bpe, and determined the crystal structure of this adduct, which is the first example of a bpe adduct of rhodium(II) acetate, as shown in Fig. 1. The adsorption property was examined for $\mathrm{N}_{2}$.

Rhodium(II) acetate was prepared by a method described in

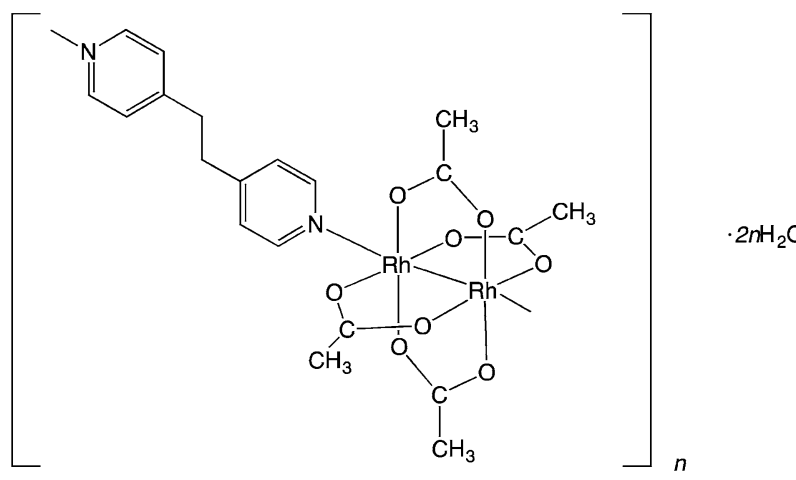

Fig. 1 Chemical structure of the title compound.

$\dagger$ To whom correspondence should be addressed. E-mail: junpei@kwansei.ac.jp the literature. ${ }^{5}$ A $6.4 \mathrm{mg}(0.035 \mathrm{mmol})$ portion of bpe was added to a solution of rhodium(II) acetate $(10.0 \mathrm{mg}, 0.0266$ $\mathrm{mmol}$ ) in $4 \mathrm{~cm}^{3}$ of ethanol, and the solution was stirred overnight. The precipitate was collected, washed by ethanol, and dried under a vacuum. Yield, $11.7 \mathrm{mg}(82 \%)$. Anal. Found: C, 38.08; H, 4.10; N, 4.58\%. Calcd for

Table 1 Crystal and experimental data

Chemical formula: $\mathrm{C}_{20} \mathrm{H}_{28} \mathrm{~N}_{2} \mathrm{O}_{10} \mathrm{Rh}_{2}$

Formula weight $=662.26$

$T=293 \mathrm{~K}$

Crystal system: monoclinic Space group: $C 2 / c$

$a=24.937(5) \AA$

$b=13.211(3) \AA$

$c=8.8480(19) \AA$

$V=2907.0(11) \AA^{3}$

$D_{\mathrm{x}}=1.513 \mathrm{~g} / \mathrm{cm}^{3}$

Radiation: Mo $K_{\alpha}(\lambda=0.71073 \AA)$

$\mu\left(\right.$ Mo $\left.K_{\alpha}\right)=1.182 \mathrm{~mm}^{-1} \quad F\left(\begin{array}{lll}0 & 0 & 0\end{array}\right)=1328$

Crystal size $=0.06 \times 0.06 \times 0.33 \mathrm{~mm}^{3}$

No. of reflections collected $=8535$

No. of independent reflections $=3324$

$\theta$ range for data collection: 1.64 to $28.43^{\circ}$

Data/Restraints/Parameters $=3324 / 24 / 181$

Goodness-of-fit on $F^{2}=0.819$

$R$ indices $[I>2 \sigma(I)]: R 1=0.0356, w R 2=0.0809$

$R$ indices (all data): $R 1=0.0921, w R 2=0.0863$

$(\Delta / \sigma)_{\max }=0.001$

$(\Delta \rho)_{\max }=0.517 \mathrm{e}^{-3} \quad(\Delta \rho)_{\min }=-0.379 \mathrm{e}^{-3}$

Measurement: Bruker Smart APEX CCD diffractometer

Program system: SHELXTL

Structure determination: Direct methods (SHELXS-97)

Refinement: full matrix least-squares (SHELXL-97)

CCDC deposition number: 921025 


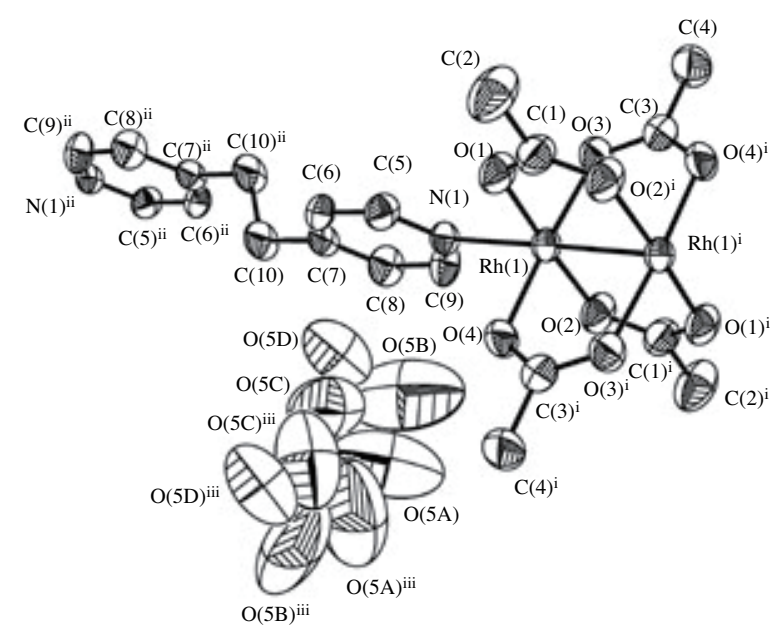

Fig. 2 ORTEP view of the title compound. Crystal water molecules are disordered into four positions with occupancy factors of 0.25. Hydrogen atoms are omitted for clarity. The thermal ellipsoids are shown at the $50 \%$ probability level. Symmetry codes: (i) $1 / 2-x, 3 / 2-y,-z$; (ii) $1-x, 1-y, 1-z$; (iii) $1-z, y, 3 / 2-z$.

$\mathrm{C}_{20} \mathrm{H}_{24} \mathrm{~N}_{2} \mathrm{O}_{8} \mathrm{Rh}_{2} \cdot 0.25 \mathrm{H}_{2} \mathrm{O}: \mathrm{C}, 38.09 ; \mathrm{H}, 3.92 ; \mathrm{N}, 4.44 \%$. IR $\left(\mathrm{KBr}, \mathrm{cm}^{-1}\right)$ : $3056(\mathrm{VCH}), 1588\left(v_{\mathrm{as}} \mathrm{COO}\right), 1429\left(v_{\mathrm{s}} \mathrm{COO}\right)$. Diffuse reflectance spectra: $\lambda_{\max } 259,329,460 \mathrm{sh}, 518\left(\pi^{*}\left(\mathrm{Rh}_{2}\right)\right.$ $\left.\rightarrow \sigma^{*}\left(\mathrm{Rh}_{2}\right)\right) \mathrm{nm}$. X-ray quality crystals were grown by the slow diffusion of bpe with rhodium(II) acetate in ethanol.

X-ray diffraction data for these crystals were collected at 293 $\mathrm{K}$ on a Bruker CCD X-ray diffractometer (SMART APEX) using graphite-monochromated Mo- $K_{\alpha}$ radiation. Crystal data and details concerning data collection are given in Table 1 . The structure was solved by direct methods and refined by fullmatrix least-squares methods. Crystal water molecules were disordered into four positions with occupancy factors of 0.25 . The hydrogen atoms, except for water molecules, were inserted at their calculated positions and fixed there. All of the calculations were carried out on a Pentium IV Windows 2000 computer utilizing the SHELXTL software package.

The molecular structure drawn by ORTEP is shown in Fig. 2. Selected bond distances and angles are given in Table 2S. The crystal structure consists of chain molecules made up of $\mathrm{Rh}_{2}\left(\mathrm{CH}_{3} \mathrm{CO}_{2}\right)_{4}$ and bpe, and water molecules. The asymmetric unit contains one-half of the dinuclear unit and bpe molecule with a crystallographic inversion center at the midpoint of the $\mathrm{Rh}(1)-\mathrm{Rh}(1)^{\mathrm{i}}$ bond and the center of the bpe moiety, respectively. The $\mathrm{Rh}(1)-\mathrm{Rh}(1)^{\mathrm{i}}$ distance is $2.3990(8) \AA$, which is in the range of $2.35-2.45 \AA$, found in the majority of dinuclear rhodium(II) carboxylates..$^{5-9}$ The coordination geometry around the rhodium atom, $\mathrm{Rh}(1)$, is a distorted octahedron. One apical position is occupied by the nitrogen atom, $\mathrm{N}(1)$, of the bpe molecule. The $\mathrm{Rh}(1)$ atom is $0.076 \AA$ above the basal plane formed by $\mathrm{O}(1)-\mathrm{O}(2)-\mathrm{O}(3)-\mathrm{O}(4)$ towards the nitrogen atom $\mathrm{N}(1)$ of the bpe molecule. The basal bond distances of the $\mathrm{Rh}$ and basal $\mathrm{O}$ atoms are 2.024(4) - 2.037(4) $\mathrm{A}$, which are comparable to those found in rhodium(II) acetate and rhodium(II) pivalate and their chain compounds. ${ }^{5-9}$ The apical $\mathrm{Rh}(1)-\mathrm{N}(1)$ distance is 2.252(4) $\AA$, which is also in the usual range as apical bonding for the rhodium(II) carboxylate adducts. ${ }^{5-9}$ The $\mathrm{Rh}(1)^{\mathrm{i}-}$ $\mathrm{Rh}(1)-\mathrm{N}(1)$ angle is $177.53(12)^{\circ}$, resulting in a zig-zag chain molecule. It is to be noted that the chain molecules are interlinked through the $\mathrm{C}-\mathrm{H} \cdots \pi$ interactions to form cavities, as shown in Fig. 3. The crystal structure is similar to that of the corresponding copper(II) species, $\left[\mathrm{Cu}_{2}\left(\mathrm{CH}_{3} \mathrm{CO}_{2}\right)_{4}(\mathrm{bpe})\right]_{n}$.

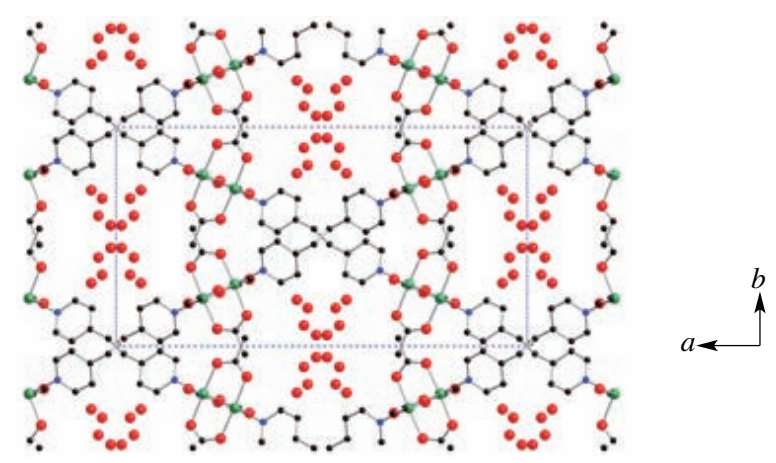

Fig. 3 Packing diagram of the title compound viewed along the $c$ axis.

$2 n \mathrm{H}_{2} \mathrm{O} .{ }^{10}$ In the crystal, water molecules are incorporated in the cavities among the chain molecules. Therefore, we expected the adsorption property to exist for the present compound, and measured the adsorption isotherm of $\mathrm{N}_{2}(77 \mathrm{~K})$. The adsorption isotherm belonged to Type II in IUPAC classification $\left(S_{\mathrm{BET}}=\right.$ $\left.65.6 \mathrm{~m}^{2} \mathrm{~g}^{-1}\right)$. The sample possessed no micropores, judging from the $t$-plot analysis (Fig. 5S), ${ }^{9}$ while the pore size distribution curve estimated from the Dollimore-Heal method ${ }^{9}$ showed that this mainly has a mesopore with a mean diameter of ca. $33 \mathrm{~nm}$ (Fig. 6S).

\section{Acknowledgements}

The present work was partially supported by MEXT-Supported Program for the Strategic Research Foundation at Private Universities and Grants-in-Aid for Scientific Research No. 22550066 from the Ministry of Education, Culture, Sports, Science and Technology (MEXT).

\section{References}

1. M. Mikuriya, D. Yoshioka, and M. Handa, Coord. Chem. Rev., 2006, 250, 2194

2. M. Mikuriya, Bull. Jpn. Soc. Coord. Chem., 2008, 52, 17.

3. R. Nukada, W. Mori, S. Takamizawa, M. Mikuriya, M. Handa, and H. Naono, Chem. Lett., 1999, 367.

4. W. Mori, H. Hoshino, Y. Nishimoto, and S. Takamizawa, Chem. Lett., 1999, 331.

5. M. Mikuriya, M. Higashiguchi, T. Sakai, D. Yoshioka, and M. Handa, in "Progress in Coordination and Bioinorganic Chemistry”, ed. M. Melnik and A. Sirota, Slovak Technical University Press, Blatislava, 2003, 213.

6. M. Mikuriya, J. Yamamoto, D. Yoshioka, H. Tanaka, and M. Handa, in "New Trends in Coordination, Bioinorganic and Applied Inorganic Chemistry", ed. M. Melnik, P. Segra, and M. Tatarko, Slovak University of Technology Press, Blatislava, 2011, 311.

7. M. Mikuriya, J. Yamamoto, D. Yoshioka, H. Tanaka, and M. Handa, X-ray Struc. Anal. Online, 2011, 27, 27

8. M. Mikuriya, J. Yamamoto, K. Ouchi, D. Yoshioka, and M. Handa, X-ray Struc. Anal. Online, 2011, 27, 69.

9. M. Mikuriya, J. Yamamoto, K. Ouchi, D. Yoshioka, H. Tanaka, and M. Handa, X-ray Struc. Anal. Online, 2013, $29,7$.

10. H.-L. Hu, M.-C. Suen, C.-W. Yeh, J.-D. Chen, Polyhedron, 2005, 24, 1497. 